\title{
¿Cómo formar en la didáctica de especialidad a futuros docentes de educación básica? Propuesta de un modelo formativo
}

How to train future education teachers in the specialty didactics?

Proposal for a model teacher formation

\author{
Rosa Gaete-Moscoso ${ }^{\mathrm{a}}$, Francisco Navia Bueno ${ }^{\mathrm{b}}$, María Alejandra Moralesc ${ }^{\mathrm{c}}$, \\ Jorge Neira Silva ${ }^{\mathrm{d}}$, Genevy Moreno Sajuria ${ }^{\mathrm{e}} \&$ Mariluz Yáñez Pérez ${ }^{\mathrm{f}}$ \\ aUniversidad Alberto Hurtado, Chile, $\measuredangle$ rgaete@uahurtado.cl \\ [orcid.org/0000-0002-2249-9338] \\ bUniversidad Alberto Hurtado, Chile, fnavia@uahurtado.cl \\ cUniversidad Alberto Hurtado, Chile, mamoralg@uahurtado.cl \\ dUniversidad Alberto Hurtado, Chile, jneira@uahurtado.cl \\ eUniversidad Alberto Hurtado, Chile, gmoreno@uahurtado.cl \\ fUniversidad Alberto Hurtado, Chile, myanez@uahurtado.cl
}

\section{RESUMEN}

Este artículo argumenta la importancia de concebir al profesor de educación general básica también como un profesor disciplinar. En el escenario actual, la política educativa señala que estos docentes deben manejar conocimientos disciplinares en nivel experto, cuestión que es problemática porque se devuelve al campo profesional como una forma de colonización de quienes son formados disciplinariamente. Ejemplificamos, mediante la exposición de elementos teóricos y curriculares de nuestro modelo de formación, un camino alternativo a esta oposición entre saberes didácticos-disciplinares y pedagógicos. Se conceptualiza la disciplina, articulada a la didáctica, como una manera de pensar el mundo. Argumentamos que la formación debe realizarse sobre la base de modelos didácticos específicos.

PALABRAS CLAVE: formación inicial docente, modelo formativo, didáctica.

\section{ABSTRACT}

This article argues the importance of conceiving the basic general education teacher as a disciplinary teacher as well. In the current scenario, education policy indicates that these teachers must handle disciplinary knowledge at an expert level, which is problematic because it is returned to the professional field as a form of colonization of those who are disciplined. 
R. Gaete-Moscoso et al.

We exemplify, by exposing theoretical and curricular elements of our training model, an alternative path to this opposition between didactic-disciplinary and pedagogical knowledge. Discipline is conceptualized, articulated with didactics, as a way of thinking about the world. We argue that the training should be carried out on the basis of specific teaching models.

KEY WORDS: Teacher formation, Model Teacher formation.

\section{INTRODUCCIÓN}

En los últimos veinte años, la profesión docente en Chile ha tenido múltiples modificaciones, lo que ha llevado a las instituciones formadoras a plantearse cambios e instalar procesos de mejora permanente. Dichos cambios han estado, además, impulsados mediante la implantación de normativas que performativizan a los programas de formación inicial (Ministerio de Educación de Chile, 2011).

Históricamente, pasado el ciclo inicial de la gran reforma educativa chilena de los años noventa, se hizo imperativo formular nuevos modelos de formación inicial docente apareciendo en la escena nacional dispositivos y programas que apuntaban a mejorar la relación entre la teoría y la práctica. Esto impulsó una formación situada, mediante el desarrollo de prácticas progresivas y con un fuerte énfasis en el conocimiento curricular vigente (Ávalos, 2002, 2004).

Desde el punto de vista teórico, esta orientación estaba basada en la crítica transversal a la excesiva teorización de la formación y, por tanto, una desvinculación sustantiva de las realidades escolares y del campo profesional en el cual las y los estudiantes de pedagogía se desempeñarían (Ávalos, 2002; Ministerio de Educación de Chile, 2005).

Dentro de los programas de formación inicial docente que surgieron en los albores de la década del 2000, nació la carrera de Educación Básica de la Universidad Alberto Hurtado, la que, respondiendo a una tradición formativa ignaciana, se sustentó en un modelo formativo en dos líneas: una formación orientada a una ampliación cultural, y una formación reflexiva que articulase teoría y práctica (Álvarez Martín, 2007). Esta segunda línea se denominó modelo de formación dual. Adecuándose a la factibilidad de su implementación y a los desafíos formativos del nuevo período social y político, este modelo sufrió una importante precisión agregando a sus dos pilares originales el favorecimiento de una formación didácticadisciplinar.

Así surgió una nueva versión del modelo formativo basada en estos tres pilares: ampliación cultural, formación reflexiva que articula teoría y práctica, y formación didáctica/ disciplinar. Este nuevo modelo tensiona dos lógicas que han estado presente en la formación docente. Por una parte, se espera que el profesor o profesora de Educación Básica desarrolle competencias de orden pedagógico transversales a todos los campos disciplinares y, por otra, se espera que el profesor o profesora de Educación Básica conozca, maneje y utilice 
los contenidos del currículum prescrito en, al menos, cuatro áreas del saber (Lenguaje y Comunicación, Ciencias Sociales, Ciencias Naturales y Matemáticas), y que dicho conocimiento sea en nivel experto para su enseñanza explícita. Estas dos lógicas quedaron plasmadas en los Estándares de Formación Inicial Docente (Ministerio de Educación de Chile, 2011) y, aunque problemáticas y cuestionadas, han sido asumidas por las instituciones formadoras que, en mayor o menor medida, buscan responder a ellas (Ávalos, 2004).

Sin embargo, un análisis de ambas lógicas nos conduce necesariamente a problematizar la formación inicial tanto en sus modelos formativos como en sus operacionalizaciones. La construcción de los planes de estudios para la formación inicial desafía a lograr, en 5 años, una formación general y una formación de especialidad que atienda a las exigencias planteadas por la normativa vigente y por la investigación realizada respecto de las competencias necesarias para el desempeño profesional en la sociedad actual (Ávalos, 2004; Ávalos et al. 2010). Dentro de este desafío, la formación didáctica y disciplinar es la que supone el mayor reto dada la amplitud del campo conceptual, curricular y didáctico de cada área, y el contraste que existe entre la formación de profesores disciplinares para enseñanza secundaria y aquellos que se desempeñan en la educación básica. La tendencia ha sido intentar trasponer un modelo disciplinar clásico en el que se estudia primeramente el contenido, coloquialmente llamado "duro", para, en un segundo momento, agregar el estudio del contenido pedagógico lo que resulta ser, generalmente, aislado del primer momento formativo.

La opción realizada por la carrera de Educación Básica de la Universidad Alberto Hurtado contraviene dicha tendencia. La opción formativa, traducida en una estructura curricular, ha sido la de diseñar asignaturas usando como base una noción de didáctica que integra el conocimiento disciplinar y el conocimiento metodológico concibiéndolo dentro de un mismo campo conceptual (Abreu et al. 2017). En consecuencia, teóricamente, esta opción ha implicado concebir y problematizar la disciplina desde sus especificidades y recortes curriculares y escolares, y mirarla con la óptica de la didáctica, haciendo un ejercicio reversible entre ambas dimensiones.

Siguiendo esa línea, cada área disciplinar ${ }^{1}$ ha optado, de manera explícita, por enseñar y utilizar un determinado modelo didáctico vigente y acorde al currículum prescrito, intencionando que las y los futuros docentes aprendan las bases conceptuales de dicho modelo, sus principios didácticos orientadores, las principales estrategias de enseñanzaaprendizaje y las lógicas en las que se construyen las secuencias didácticas. Esta formación disciplinar se coordina tanto con una formación explícita en temas de orden pedagógico tales como el diseño de experiencias educativas, la evaluación pedagógica y la teoría curricular, así como una formación explícita y transversal de competencias reflexivas, de investigación didáctica y de aprendizaje experiencial mediante Experiencias Laborales y Prácticas.

La carrera forma en cuatro áreas didácticas/disciplinares: Lenguaje y Comunicación, Matemáticas, Ciencias Naturales y Ciencias Sociales. 
R. Gaete-Moscoso et al.

Asumiendo el consenso sobre la importancia de la formación disciplinar en la profesión de educación básica, este artículo busca exponer y discutir las opciones formativas de trabajar desde una noción de disciplina escolar que reconoce la didáctica como parte integral de la misma, distinguiéndola de la disciplina teórica y alejándose de concepciones lineales de la formación en la misma. Se busca, mediante esta reflexión, avanzar en responder a la pregunta ¿cómo formar un profesor de educación básica, disciplinariamente, atendiendo, al mismo tiempo, la compleja relación que existe, en el campo laboral, entre la enseñanzaaprendizaje y sus variables intervinientes?

Esta sistematización surge del análisis y evaluación de las herramientas curriculares del programa antes mencionado, trabajo que fue desarrollado durante los años 2014-2018 por el equipo curricular de este programa, en el contexto de la implementación del Proyecto de Mejora Institucional (PMI $1501 \mathrm{UAH}$ ). Esta revisión consideró el trabajo con fuentes escritas: (i) el proyecto original de la carrera, (ii) la propuesta de modificación implementada a partir del año 2012, (iii) los actuales programas de las asignaturas que componen el plan de estudios y (iv) literatura especializada.

Se concluye que fortalecer una formación didáctica específica y disciplinar no se contrapone a una formación pedagógica en la medida que las bases teóricas sean coherentes entre sí, y que los dispositivos de formación tengan una orientación explícita hacia la articulación de todas las dimensiones del saber profesional docente (Tardif, 2004; Shulman, 2005). La coherencia y la articulación de saberes, en particular los didácticos-disciplinares, estarían aseguradas, entre otros aspectos, por la asunción explícita de modelos didácticos en cada mención que operarían como objeto y medio de conocimiento.

\section{LA PROPUESTA FORMATIVA EN SU CONJUNTO: TRES PILARES}

La carrera de Educación Básica desarrolla una formación que considera tres ámbitos: formación con orientación a la ampliación cultural, formación en reflexividad que articula teoría y práctica, y formación en didáctica específica.

Estos tres ámbitos se abordan, curricularmente, en dos ciclos formativos: un ciclo básico de cuatro semestres y un ciclo de especialización de seis.

En el primer ciclo, se busca que el futuro docente desarrolle competencias genéricas (comunicativas y de razonamiento matemático) y amplíe su horizonte cultural mediante la formación en áreas humanistas como filosofía, historia, ciencias y arte. A estos dos grandes objetivos formativos se agrega una línea de introducción al campo del conocimiento pedagógico y a ciertos conocimientos generales de las disciplinas.

El segundo ciclo, de especialización, considera el desarrollo de competencias profesionales en saberes pedagógicos (como diseño de experiencias educativas, evaluación, etc.) y en saberes didácticos/disciplinares, los cuales se abordan en dos dispositivos de suma relevancia: la formación académica y la formación en la práctica. 
La formación en la práctica contempla la inserción temprana en establecimientos educativos, con el acompañamiento de un profesor guía (docente de aula) y de un profesor tutor (académico universitario). En el acompañamiento de este último, se trabaja la reflexión sobre la práctica, la cual es imprescindible para articular teoría y práctica (Álvarez Martín, 2007). Deberá entenderse que la Práctica hace parte de la formación didáctica-disciplinar y es la última fase de la formación en la práctica que se inaugura en el $5^{\circ}$ semestre de la carrera con las Experiencias Laborales (ELAB), y es el dispositivo de cierre que articula los aprendizajes anteriores de ELAB, de los cursos en Didáctica y de los cursos de la línea Pedagógica (Fig. 1).

Este modelo general de formación tiene un ámbito específico de la formación en didáctica de la especialidad cuyo primer escollo a resolver es el concepto de la didáctica. Este primer problema se aborda mediante el análisis de un aspecto común a las didácticas específicas que, actualmente, son reconocidas por el currículum escolar y vigentes en el mundo de la investigación especializada, a saber: el constructivismo.

\section{CONSTRUCTIVISMO Y MODELOS DIDÁCTICOS DE ENSEÑANZA-APRENDIZAJE DE CUATRO DISCIPLINAS DEL CURRÍCULUM PRESCRITO. ELEMENTOS COMUNES A LAS DIDÁCTICAS ESPECÍFICAS ACTUALES}

El constructivismo y sus derivaciones (Ausubel et al. 1983; Baquero, 1997; Vygotski, 1995, 1996; Rosas \& Sebastián, 2001) son, en la actualidad, un tronco conceptual común de las didácticas específicas que se estudian tanto en Chile como en diversos países.

Es el caso del enfoque comunicativo promovido en la didáctica del lenguaje oral y escrito (Maqueo, 2006; Lomas et al. 2015), del enfoque del aprendizaje por descubrimiento utilizado en la didáctica de la Historia, Geografía y Ciencias Sociales (Santisteban \& Pagès, 2011; Sallés Tena \& Santacana Mestre, 2016), del enfoque indagatorio propio de las Ciencias Naturales (Harlen, 1998) y del enfoque de resolución de problemas o la Teoría de Situaciones Didácticas (Brousseau, 2007).

Cada uno de estos enfoques didácticos opera con, al menos, tres grandes principios derivados de los aportes del constructivismo: (i) concepción del contenido a enseñar, (ii) concepción de quien aprende y de quien enseña y (iii) forma en que debe organizarse la enseñanza y aprendizaje, los que revisaremos a continuación.

\section{El conocimiento como un saber contextualizado}

Los modelos didácticos anteriormente enumerados parten del supuesto que lo que se enseña es un corpus de conocimiento que tiene una naturaleza histórica, por tanto, cambiante en el tiempo y socialmente construida. De allí que cualquier contenido de la enseñanza deba ser entendido en relación a su contexto, considerando sus condiciones de producción y problematizándolo desde las apuestas ideológicas que lo llevaron a su supremacía. 
R. Gaete-Moscoso et al.

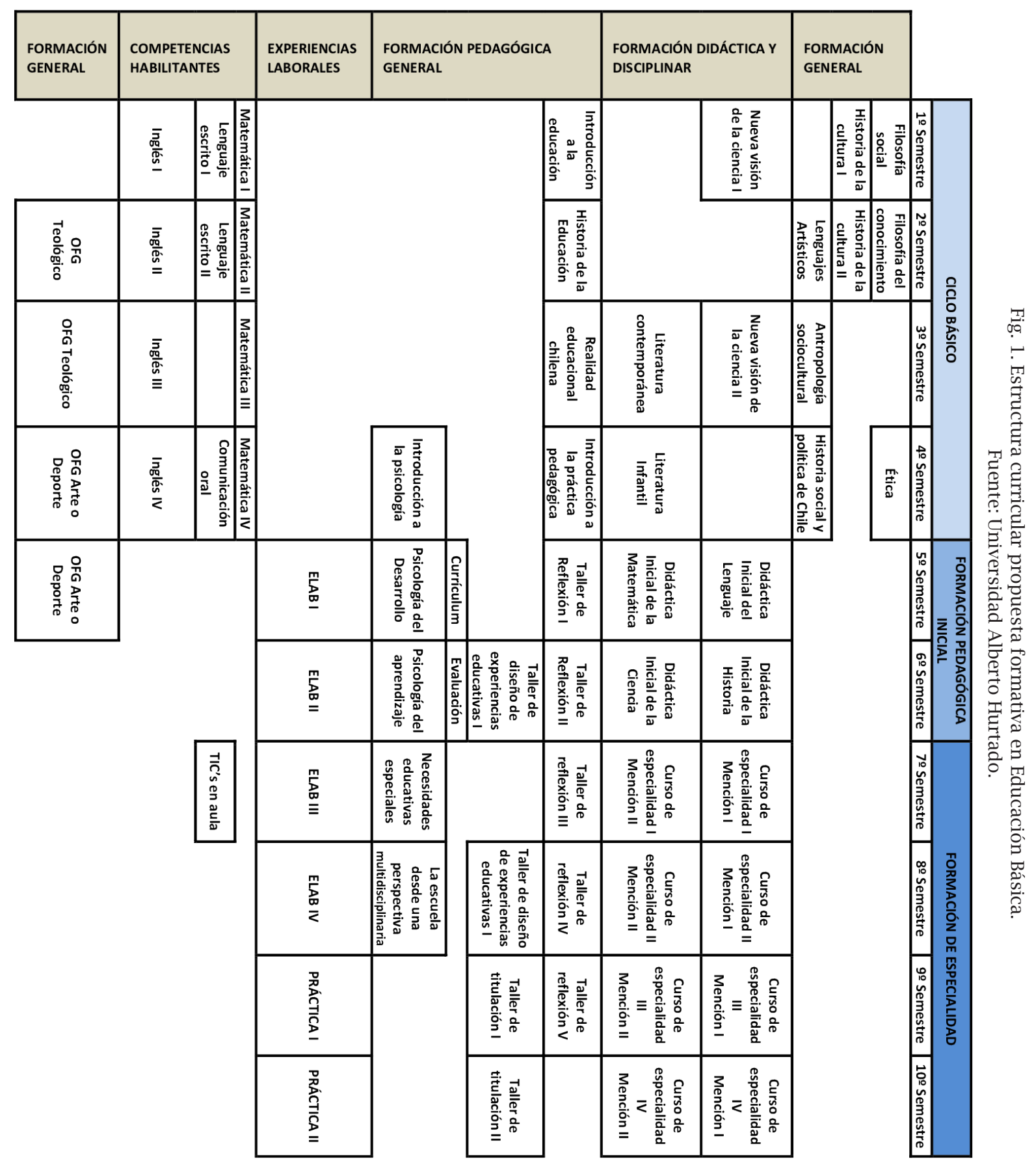


Esto desafía al docente a estudiar el contenido conceptual con una actitud de sospecha epistémica permanente sin por ello asumir, necesariamente, una postura postmodernista o deconstruida, sino comprendiendo su naturaleza evolutiva y de respuesta parcial a los problemas abordados por una determinada disciplina respecto de un determinado fenómeno y en un determinado momento histórico.

En esta comprensión, la formación inicial debiese proveer oportunidades para que el futuro docente estudie el contenido conceptual contrastando distintas ópticas y reflexionando críticamente sobre cada una de ellas. El fruto de esta reflexión debiese conducirlo a comprender que su mandato social de enseñar los contenidos curricularmente establecidos no anula su postura crítica frente a ellos. Por esta vía, un modelo formativo que considere esta concepción debiese impulsar a que las y los futuros profesores adopten una perspectiva fundamentada respecto de lo que van a enseñar.

Que el conocimiento sea contextualizado implica también comprender que debe ser recreado en función del contexto específico en el cual será enseñado. De allí que desde la preparación de la enseñanza, en estos modelos didácticos se espera que el docente conozca quiénes son los y las estudiantes a quienes estará dirigida la enseñanza, comprenda las claves culturales en las que se desenvuelven, relacione tales claves culturales con el contenido escolar que se enseñará, conozca cuál es la historia de aprendizaje que tienen dichos estudiantes, etc., de manera que dicha preparación tenga como producto un diseño de experiencias educativas que incorpore la variabilidad y multifactorialidad del aprendizaje, relacionando el contexto cultural en el cual se desarrollará la enseñanza con el saber establecido en el currículum prescrito y en la disciplina correspondiente.

Esto implicaría implementar una formación inicial que provea a las y los futuros docentes de oportunidades de conocimiento de contextos reales en los cuales puedan problematizar el conocimiento teórico respecto de las personas reales que se enfrentarán a su aprendizaje. Las y los estudiantes de pedagogía se verán entonces interpelados a transitar en, al menos, dos ámbitos de conocimiento: conocimiento del saber disciplinar y sus condiciones de producción, conocimiento de sus estudiantes y del micro y macro contexto en el cual viven y se desenvuelven. La manera de resolver la distancia entre ambos ámbitos será a través del diseño de experiencias educativas y de la implementación de las mismas.

\section{El aprendiz y el educador como sujetos activos y situados}

Respecto de quién aprende y de quién enseña, estos modelos didácticos reconocen al sujeto “cognitivo", constructor de sus aprendizajes (Rosas \& Sebastián, 2001). Simplificando esta idea, esto equivale a decir que se trata de personas activas, con agencia, con historia y que viven en determinados contextos que han incidido en sus trayectorias de aprendizaje (Sebastián, 2005) y que, por tanto, han desarrollado determinadas actitudes frente a los contenidos escolares que deben aprender y enseñar. 
R. Gaete-Moscoso et al.

En este sentido, al igual que respecto del conocimiento disciplinar, los individuos que participan del espacio de enseñanza-aprendizaje se encuentran situados en el tiempo y en el espacio, y participan de una determinada comunidad que, en parte, les determina aunque no los limita. Para el docente, esto implica reconocer el acervo cultural y los aprendizajes previos de sus estudiantes, así como su propia trayectoria de aprendizaje. Este reconocimiento contiene una doble dimensión: una de carácter filosófica y humanista, y otra de carácter técnico.

En su dimensión humanista, el reconocimiento del otro se traduce en una actitud de empatía, de curiosidad por quienes llegan al encuentro en la situación de aprendizaje. Es otorgar un estatus de persona y de identidad que implica respeto por ese que aprende. En su dimensión técnica, el docente debe manejar estrategias que le permitan conocer qué saben sus estudiantes, de dónde provienen, cuál es su historia, etc. Estas estrategias se traducen en actividades que se usan en los dispositivos de aprendizaje y que, por tanto, deben ser consideradas en la planificación de aula.

De este modo, en la formación inicial se debe proveer de oportunidades para que las y los futuros docentes construyan este sentido humanista y, al mismo tiempo, conozcan formas y medios para el reconocimiento del otro en todas sus dimensiones y, en particular, en su dimensión cognoscitiva.

\section{La actividad mediada como forma privilegiada de construir el aprendizaje}

El fundamento principal de los modelos didácticos es que el aprendizaje debe transitar desde la experiencia hacia la formalización del saber, reconociendo los saberes o aprendizajes previos de las y los estudiantes (Miras, 1998) y estableciendo relaciones explícitas entre dichos aprendizajes y el conocimiento establecido en los currículums. De allí que no sea extraño que las propuestas de las didácticas específicas contemplen trabajar con preguntas problematizadoras o situaciones problemáticas provenientes de la experiencia cotidiana (Ciencias Sociales, Ciencias Naturales y Matemáticas) o problemas comunicativos reales (Lenguaje y Comunicación). Estos puntos de partida, además, se deben articular en estructuras comunes que provienen, precisamente, de unas formas discursivas más o menos establecidas (Mehan, 1979) como, por ejemplo, la estructura de la clase que comienza con la activación de conocimientos previos y culmina con la sistematización de saberes mediante procesos metacognitivos.

En el enfoque constructivista, la organización de la secuencia antes señalada (de la experiencia hacia la formalización) requiere de formas específicas de implementarla, las que se conciben como formas de interacción didáctica (Villalta, 2009; Villalta \& Martinic, 2009).

La interacción didáctica, que responda a una forma constructivista de concebir y realizar la enseñanza, considera la acción de quien enseña en lógica de mediación. Así, el docente debiese proveer situaciones de aprendizaje a sus estudiantes incitándolos, y hasta 
cierto punto impulsándolos, a la construcción del conocimiento desde la experiencia hasta la formalización de dicho conocimiento.

Todos los modelos didácticos vigentes consideran la enseñanza y el aprendizaje como un proceso de mediación (Wertsch, 1999; Baquero, 2009). En una primera acepción, la mediación es entendida, teóricamente, como una práctica intencionada, por parte de quien enseña, que facilita el acceso a un saber mediante el establecimiento de puentes entre dicho saber y los aprendizajes previos de quien aprende. Estos puentes deben ser explícitamente diseñados por quien enseña (incluyendo pares aventajados) e, independientemente de la existencia de mediaciones de carácter fortuito, debiesen tener como efecto una modificación en la estructura de conocimiento de quien aprende (Ausubel et al. 1983), es decir, debiesen trabajar en (activar) la Zona de Desarrollo Próximo (Vygotski, 1996; Baquero, 1997).

Pero la actividad de mediación no son solo esos puentes, es decir, no es solo una acción auxiliar para desarrollar unas determinadas competencias, sino que es, al mismo tiempo, una particular manera de interactuar en el mundo, usando sistemas de signos y transformándose gracias a ellos. En esta lógica, una disciplina constituye un sistema de signos, un determinado lenguaje con el cual comprendemos, abordamos y vivimos la realidad material y psicológica, pero que, al mismo tiempo, nos modifica sustantivamente. Así, el aprendizaje de una determinada disciplina es, al mismo tiempo, aprender su código y aprender una manera específica de comprender la realidad. En este sentido, como señala Baquero (2009), los instrumentos de mediación no son meros auxiliares de la acción humana, sino que la transforman, lo que para el caso de las disciplinas escolares no es únicamente el corpus conceptual el que constituye este sistema de signos, sino también las herramientas que se ponen en uso, las cuales están constantemente atravesadas por formas de interacción (discursos) específicas a la escuela (Wertsch, 1999). El sistema de signos, así constituido, no es la disciplina entendida en el sentido clásico, sino un nuevo corpus en el que se articulan estas tres dimensiones.

En consecuencia, la preparación de un docente de educación básica en el ámbito disciplinar implicaría que aprendiese determinadas herramientas de mediación, es decir, maneras específicas de pensar y de enseñar a otros a pensar de tales maneras. Por tanto, el futuro profesor debiese aprender estas formas específicas de estructurar su propio pensamiento y a desarrollar interacciones didácticas mediante las cuales dicho tipo de pensamiento pueda ser enseñado a otros.

\section{PROPUESTA FORMATIVA EN DIDÁCTICA DE LA ESPECIALIDAD}

Como ya se aventuró al comienzo de esta reflexión, la tensión entre una formación generalista y una formación de especialidad ha contrapuesto dos campos, el pedagógico y el didáctico-disciplinar, como si se tratase de ámbitos totalmente diferenciados. En este sentido, la propuesta formativa para el ámbito de la formación didáctica/disciplinar 
considera dos elementos: (i) los principios constructivistas comunes a los modelos didácticos de enseñanza/aprendizaje de las disciplinas escolares; y (ii) la conceptualización de las disciplinas escolares como un campo de conocimiento ligeramente distinto a la concepción clásica. Estos dos elementos se plasman en dos principios formativos: (i) uso del modelo didáctico imperante como medio y objeto de conocimiento y (ii) una estructura curricular coherente, temáticamente, con el currículum escolar (por tanto, secuenciada en temas disciplinares, los cuales, valga la redundancia, son abordados mediante el modelo didáctico de cada disciplina) (Fig. 2).

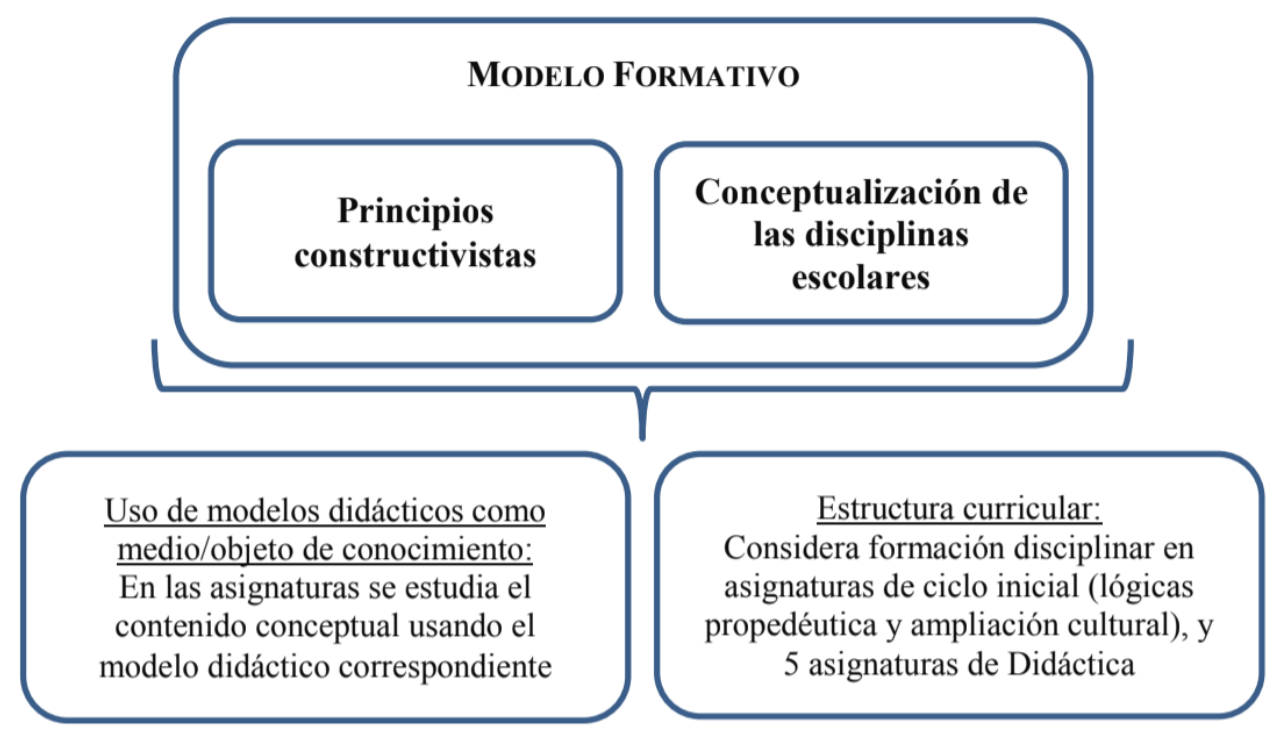

Fig. 2. Modelo formativo en la especialidad. Fuente: Elaboración propia.

La apuesta es formar didáctica y disciplinarmente en torno al aprendizaje de un modelo didáctico, el cual es, al mismo tiempo, objeto y medio de conocimiento. Así, el abordaje de los contenidos de formación se realiza siguiendo los mismos principios y las mismas estrategias propuestas en cada modelo.

A modo de ejemplo, en Lenguaje y Comunicación se utilizan textos auténticos, se parte del acervo cultural del estudiante reconociendo sus aprendizajes previos (que incluye su desarrollo lector y escritor), se trabaja con estrategias cognitivas y metacognitivas, se proponen tareas comunicativas auténticas. Todo ello mientras se enseña literatura, gramática, producción de textos, etc. Coherentemente, en Ciencias Naturales se proponen experiencias prácticas de indagación para aprender Biología, Física y Química.

La estructura curricular propuesta contempla una asignatura de Didáctica Inicial y cuatro asignaturas de Didáctica de la Especialidad, más un trabajo de fin de estudios y una práctica final.

En Didáctica Inicial, las y los estudiantes abordan el conocimiento del enfoque y los modelos didácticos (en algunos casos se trata de un modelo único) vigentes y coherentes 
con el enfoque señalado. Este abordaje incluye el aprendizaje de los principios teóricos generales, las principales estrategias y la adecuación de las mismas a los niveles iniciales de la escolaridad. Al igual que en las fases siguientes, la programación de clases incluye momentos de trabajo teórico, trabajo práctico y reflexión o metacognición de lo aprendido. Este aprendizaje se desarrolla en articulación con el conocimiento curricular y de evaluación, específico y general, y con el aprendizaje del modelo de planificación en Unidades Didácticas Integradas (Gaete-Moscoso, 2013).

La segunda etapa es la formación en didáctica de especialidad en relación a las etapas intermedias y avanzadas de la escolaridad. La opción fue organizar cuatro asignaturas con cuatro áreas temáticas (coincidentes con los ejes del currículum escolar en cada área), en las que, nuevamente, se trabaja el aprendizaje del conocimiento disciplinar mediante la implementación y la reflexión sobre un determinado modelo didáctico. Para ello, los y las estudiantes deben realizar experiencias concretas, diseñar otras para ser implementadas ${ }^{2} y$, constantemente, reflexionar sobre lo que están aprendiendo, sistematizando el contenido disciplinar. La diferenciación en áreas temáticas posibilita ampliar la cobertura del contenido disciplinar estudiado. La reflexión sobre la experiencia permite vincular el conocimiento teórico y el conocimiento experiencial como profesor en formación con la Experiencia Laboral realizada.

La última etapa es la realización de un trabajo de título y una práctica profesional. Ambos elementos son los espacios privilegiados para el trabajo de resignificación final de la trayectoria realizada en las dos etapas anteriores. Así, la sistematización teórica didácticadisciplinar se coordina con la pedagógica y se plasma en un diseño que, obligatoriamente, debe ser implementado. El espacio de la práctica es el momento de intencionar y probar experiencias docentes nuevas y desafiantes en lógica de investigación didáctica, es decir, realizando un levantamiento de un problema didáctico en contexto real, diseñando una propuesta formativa y evaluando los aprendizajes obtenidos por los estudiantes después de la implementación. Esto culmina con la reflexión sobre los aprendizajes profesionales desarrollados a lo largo del período.

Además de la estructura curricular y las intenciones movilizadas en ella, un segundo aspecto es el diseño de las programaciones específicas en cada asignatura.

En relación a este segundo aspecto, el supuesto de trabajo es que el futuro docente podrá modificar sus formas de enseñanza si (i) lo engarza a su conocimiento anterior, (ii) lo experimenta (lo vivencia y lo ensaya con otros) y (iii) lo reflexiona. De allí que, en las asignaturas de didáctica, se intencionan experiencias prácticas de todo tipo, además de las propias de la ELAB: laboratorios, salidas a terreno, talleres prácticos para diseñar experiencias y talleres prácticos para probar en ellos mismos las distintas estrategias.

Para ello, los formadores realizan técnicas, siendo las más privilegiadas las denominadas "activo-participativas" (Vargas Vargas \& Bustillos de Núñez, 1992, 1998;

2 En algunos casos se logra realizar la implementación de la planificación. 
R. Gaete-Moscoso et al.

Hourst \& Sivasailam, 2005), las que son diseñadas de acuerdo a los modelos didácticos enunciados al inicio de este artículo, es decir, considerando los principios y secuencias didácticas de los mismos.

Para el caso de Matemáticas es un proceso que va desde el planteamiento de situaciones problemáticas a la formalización del conocimiento. En el de Ciencias Naturales, siguiendo un modelo indagatorio, simula el trabajo científico desde la formulación de un problema basado en la observación de un fenómeno natural a la constitución del conocimiento científico formal. En el campo de las Ciencias Sociales, se trabaja en la lógica del rol de quien hace y es partícipe de la historia, desarrollando competencias y habilidades relacionadas al pensamiento histórico, el dispositivo de aprendizaje incluye el estudio y análisis de fuentes como medio privilegiado de problematización del contenido disciplinar. Por último, en Lenguaje y Comunicación, el trabajo se organiza en torno al modelo comunicativo de enseñanza y aprendizaje, contemplando problemas comunicativos reales ${ }^{3}$ y aprendiendo estrategias procesuales (como los Tres momentos de la lectura o los Momentos de la producción escrita) y estrategias puntuales para la enseñanza y el aprendizaje del lenguaje oral y escrito.

Un elemento que no podemos dejar de mencionar es la forma de evaluar el desempeño del futuro docente y la reflexión que sobre aquello se hace. Siguiendo un modelo de evaluación auténtica (Condemarín \& Medina, 2000), los dispositivos de evaluación son connaturales al proceso de enseñanza y aprendizaje, por tanto, se diseñan e implementan en sintonía y sincronía con las actividades formativas. Es por ello que los productos de tales actividades pueden ser sujetos de calificación mediante el uso de pautas y rúbricas elaboradas sobre criterios conocidos previamente por las y los futuros docentes. El resultado es comunicado y reflexionado conjuntamente con las y los estudiantes en dos niveles: la identificación de sus fortalezas y debilidades en el desarrollo de la tarea (que puede incluir el análisis de su manejo conceptual y teórico), y la proyección de la forma en que fue evaluado hacia la forma en que evaluará a sus futuros estudiantes.

$\mathrm{Al}$ integrar el rescate de la experiencia como una forma de acceso al conocimiento, la conducción académica del proceso formativo desafía a formular un modelo que debe, imperativamente, contemplar tres grandes dimensiones: el concepto de lo que se va a enseñar, el concepto de quién aprende y de quién enseña, y el concepto de cómo se va a enseñar.

El modelo formativo en didáctica específica, en suma, trabaja sobre los siguientes principios:

- El reconocimiento del capital cultural que tiene el futuro profesor.

- Reflexión permanente sobre lo que se aprende y enseña reconociendo sus contextos de producción.

3 En el caso de las y los estudiantes en formación docente incluye, por ejemplo, problemas reales de su desempeño académico, como escribir reportes de investigación, hacer informes didácticos y pedagógicos, desarrollar planificaciones o diseño de experiencias de aprendizaje, elaborar manuales didácticos. Esto último es también una actividad que se realiza en las otras áreas didácticas-disciplinares. 
¿CÓMO FORMAR EN LA DIDÁCTICA DE ESPECIALIDAD A FUTUROS DOCENTES DE EDUCACIÓN BÁSICA?

- Una organización curricular que articula el conocimiento didáctico-disciplinar con el currículum escolar.

- Una implementación del currículum que considera la experiencia del futuro docente como el puntapié inicial del proceso de aprendizaje.

\section{CONCLUSIÓN: LOS DESAFÍOS EN ELABORACIÓN DE PROGRAMAS DE FORMACIÓN INICIAL DE PROFESORES Y PROFESORAS DE EDUCACIÓN BÁSICA.}

Evidentemente encontrar una concordancia entre lo que se declara y lo que finalmente se hace es una de las tareas más complejas del quehacer pedagógico.

El modelo que hemos desarrollado en la carrera de Educación Básica está en constante cuestionamiento tanto por los actores involucrados, como por las señales que el sistema educativo envía a todos los programas formativos.

Obviando esas tensiones, no por desear ignorarlas sino porque son materia de un análisis distinto, queremos ofrecer esta sistematización de nuestro modelo concluyendo con una serie de reflexiones que podremos ahondar en etapas futuras de este trabajo.

En primer lugar, el trabajo conceptual sobre el aprendizaje de una profesión ha estado subsumido a la discusión por las condiciones organizacionales y políticas sobre las cuales desarrollar la formación docente (Agencia de Calidad de la Educación, 2016). Los avances más significativos han estado en torno a la importancia de desarrollar la formación inicial como una experiencia situada y en la práctica, pero todavía falta estudiar sobre los modos en que se desarrolla la transformación de un estudiante que desea ser profesor y la de un egresado que comienza a desempeñarse como tal.

El modelo formativo aquí presentado es un esbozo de avance en esta línea, pero, ciertamente, tiene la debilidad de surgir de una experiencia idiosincrática y, por tanto, específica, la que esperamos sirva de inspiración a otros equipos que se encuentran en esta misma labor formativa. Queda desarrollar un dispositivo de investigación que analice los elementos aquí señalados como supuestos de trabajo, de manera de densificar el dato inicial que congrega los aportes teóricos del constructivismo y de los modelos didácticos actualmente validados por las distintas comunidades científicas y académicas.

En segundo lugar, la propuesta curricular aquí detallada tiene algunas desventajas propias de los currículums organizados por asignaturas, cuya principal debilidad es la tendencia a desagregar el saber y, en ello, desproveer a los aprendices de oportunidades de construcción de sentido de sus trayectorias formativas (Torres Santomé, 2012). Es decir, fomenta el rol estudiantil clásico, centrado en el logro de resultados y en la consecución de etapas mediante comportamientos estratégicos, en desmedro de la construcción identitaria del docente en formación, por tanto, comprometido con el aquí y ahora que mira el futuro no como la meta a lograr sino como un momento de término de una formación que deberá, necesariamente, continuar durante toda su vida profesional. 
R. Gaete-Moscoso et al.

En tercer lugar, la implementación de esta propuesta formativa choca con la cultura escolar aprendida, esbozada en el párrafo anterior. Las y los académicos colisionan regularmente con algunas actitudes estudiantiles y propias, que otorgan valor supremo al conocimiento disciplinar clásico y al rendimiento académico manifestado en calificaciones. Esta tensión se ve agudizada ante las evaluaciones estandarizadas que deben presentar las y los futuros docentes. La realización de actividades inspiradas en metodologías activas y participativas, la centralidad de los espacios para la reflexión, la discusión acerca de los sentidos y fines de lo que se hace, etc., atentan contra la cobertura del contenido, provocando contradicciones vitales en los académicos como en los estudiantes.

Queda mantener la vigilancia epistémica sobre lo que se hace y sobre los fundamentos de este trabajo formativo, considerando que, al igual como lo fomentamos en nuestros futuros docentes, la reflexión permanente y crítica parece ser el único camino constante para auto-observarse e introducir, consistente y coherentemente, los cambios imprescindibles para mejorar esta noble tarea.

\section{REFERENCIAS}

Abreu, O., Gallegos, M. C., Jácome, J. G., \& Martínez, R. J. (2017). La Didáctica: Epistemología y Definición en la Facultad de Ciencias Administrativas y Económicas de la Universidad Técnica del Norte del Ecuador. Formación Universitaria, 10(3). http://dx.doi. org/10.4067/S0718-50062017000300009

Agencia de Calidad de la Educación. (2016). Estudios sobre Formación Inicial Docente (FID) en Chile. Santiago de Chile: Agencia de Calidad de la Educación.

Álvarez Martín, F. (2007). Tendencias y desafíos en la formación de docentes (Descripción de un modelo). Foro Educacional, 12, 53-68.

Ausubel, D. P., Novak, J. D., \& Hanesian, H. (1983). Psicología educativa: Un punto de vista cognoscitivo. México D.F.: Trillas.

Ávalos, B. (2002). Profesores para Chile: Historia de un proyecto. Santiago de Chile: Ministerio de Educación.

Ávalos, B. (2004). Las instituciones formadoras de docentes y las claves para formar buenos docentes. Santiago de Chile: Ministerio de Educación de Chile.

Ávalos, B., Cavada, P., Pardo, M., \& Sotomayor, C. (2010). La profesión docente: Temas y discusiones en la literatura internacional. Estudios pedagógicos (Valdivia), XXXVI(1), 235-263. https://doi. org/10.4067/S0718-07052010000100013

Baquero, R. (1997). La Zona de Desarrollo Próximo y el análisis de las prácticas educativas. En Vigotsky y el aprendizaje escolar. Buenos Aires, Argentina: Aique.

Baquero, R. (2009). Vigotsky y el aprendizaje escolar. Buenos Aires, Argentina: Aique.

Brousseau, G. (2007). Iniciación al estudio de la teoría de las situaciones didácticas. Buenos Aires, Argentina: Editorial Libros del Zorzal. 
¿CÓMO FORMAR EN LA DIDÁCTICA DE ESPECIALIDAD A FUTUROS DOCENTES DE EDUCACIÓN BÁSICA?

Condemarín, M., \& Medina, A. (2000). Evaluación auténtica de los aprendizajes: Un medio para mejorar las competencias en Lenguaje y Comunicación. Santiago de Chile: Editorial Andrés Bello.

Gaete-Moscoso, R. (2013). Elaborando una unidad didáctica. Cuaderno de Educación 54. Facultad de Educación Universidad Alberto Hurtado. Santiago de Chile. Recuperado de http://mailing.uahurtado.cl/cuaderno_educacion_54/pdf/articulo\%2054.pdf

Harlen, W. (1998). Enseñanza y aprendizaje de las ciencias. Madrid: Ediciones Morata.

Hourst, B., \& Sivasailam, T. (2005). Modèles de jeux de formation. Les jeux-cadres de Thiagi. Paris: Éditions d'Organisation.

Lomas, C., Bronckart, J.-P., Colomer, T., Gracida, Y., Jover, G., Martínez Montes, G. T., López Villaba, M.A., \& Tusón, A. (2015). Fundamentos para una enseñanza comunicativa del lenguaje. Barcelona: GRAÓ.

Maqueo, A. M. (2006). Lengua, aprendizaje y enseñanza. El enfoque comunicativo: De la teoría a la práctica. México D.F.: Limusa Editores.

Mehan, H. (1979). The structure of classroom lessons. En Learning Lessons: Social organization in the classroom. Cambridge, Massachusetts: Harvard University Press.

Ministerio de Educación de Chile. (2005). Informe Comisión sobre Formación Inicial Docente. Santiago de Chile: Ministerio de Educación de Chile.

Ministerio de Educación de Chile. (2011). Estándares orientadores para egresados de carreras de pedagogía en Educación Básica. Santiago de Chile: Ministerio de Educación - LOM Ediciones.

Miras, M. (1998). Un punto de partida para el aprendizaje de nuevos contenidos: Los conocimientos previos. En C. Coll, E. Martín, T. Mauri, J. Onrubia, I. Solé y A. Zabala. El constructivismo en el aula. Barcelona: GRAÓ.

Rosas, R., \& Sebastián, C. (2001). Piaget, Vigostki y Maturana. Constructivismo a tres voces. Buenos Aires, Argentina: Aique.

Sallés Tena, N., \& Santacana Mestre, J. (2016). Los grupos de innovación educativa en la Enseñanza de la Historia en España: Análisis póstumo de los resultados de la aplicación del método por descubrimiento y estado de la cuestión de los aprendizajes por descubrimiento. Educatio Siglo XXI, 34(2), 145-166.

Santisteban, A., \& Pagès, J. (2011). Didáctica del Conocimiento del Medio Social y Cultural en la Educación Primaria. Madrid: Síntesis.

Sebastián, C. (2005). Trajectoires de changement dans la formation d'adultes. Relations entre dynamiques identitaires, engagement et apprentissage dans le cadre d'une formation en alphabétisation (Doctorado). Université Catholique de Louvain, Louvain-la-Neuve, Bélgica.

Shulman, L. S. (2005). El saber y entender de la profesión docente. Estudios públicos (99), 195224.

Tardif, M. (2004). Los saberes del docente y su desarrollo profesional. Madrid: Narcea Ediciones. 
R. Gaete-Moscoso et al.

Torres Santomé, J. (2012). Globalización e interdisciplinariedad: El currículum integrado. Madrid: Ediciones Morata.

Vargas Vargas, L., \& Bustillos de Núñez, G. (1992). Técnicas participativas para la educación popular 2. Santiago de Chile: Argé Limitada.

Vargas Vargas, L., \& Bustillos de Núñez, G. (1998). Técnicas participativas para la educación popular. Santiago de Chile: LOM Ediciones.

Villalta, M. (2009). Análisis de la conversación. Una propuesta para el estudio de la interacción didáctica en la sala de clases. Estudios pedagógicos (Valdivia), XXXV(1), 221-238.

Villalta, M., \& Martinic, S. (2009). Modelos de estudio de la interacción didáctica en la sala de clases. Investigación y Postgrado, 24(2), 61-76.

Vygotski, L. S. (1995). Pensamiento y lenguaje. Barcelona: Paidós.

Vygotski, L. S. (1996). El desarrollo de los procesos psicológicos superiores. Barcelona: Crítica.

Wertsch, J. (1999). La mente en acción. Buenos Aires, Argentina: Aique. 